\title{
Overview of the J-TEXT progress on RMP and disruption
}

\section{physics}

Yonghua DING (丁永华) ${ }^{1}$, Zhongyong CHEN (陈忠勇) $)^{1, *}$, Zhipeng CHEN (陈志鹏) ${ }^{1}$, Zhoujun YANG (杨州军) $)^{1}$, Nengchao WANG (王能超 $)^{1}$, Qiming HU (胡启明) $)^{1}$, Bo RAO (饶波 $)^{1}$, Jie CHEN (陈杰) $)^{1}$, Zhifeng CHENG (程芝峰) $)^{1}$, Li GAO (高丽) $)^{1}$, Zhonghe JIANG (江中和) ${ }^{1}, \mathrm{Lu}$ WANG (王璐) $)^{1}$, Zhijiang WANG (王之江) ${ }^{1}$, Xiaoqing ZHANG (张晓卿) ${ }^{1}$, Wei ZHENG (郑玮) ${ }^{1}$, Ming ZHANG (张明) ${ }^{1}$, Ge ZHUANG (庄革) ${ }^{1}$, Qingquan YU (虞清泉) ${ }^{2}$, Yunfeng LIANG (梁云 峰) $)^{1,2,3}$, Kexun YU (于克训) $)^{1}$, Xiwei HU (胡希伟) ${ }^{1}$, Yuan PAN (潘垣) $)^{1}$, Kenneth William GENTLE $^{5}$ and the J-TEXT Team

${ }^{1}$ International Joint Research Laboratory of Magnetic Confinement Fusion and Plasma Physics, State Key Laboratory of Advanced Electromagnetic Engineering and Technology, School of Electrical and Electronic Engineering, Huazhong University of Science and Technology, Wuhan, 430074, People's Republic of China

${ }^{2}$ Max-Planck-Institut für Plasmaphysik, 85748 Garching, Germany

${ }^{3}$ Forschungszentrum Jülich GmbH, Institut für Energie-und Klimaforschung-Plasmaphysik, 52425 Jülich, Germany

${ }^{4}$ Institute of Plasma Physics, Chinese Academy of Sciences, Hefei 230031, People's Republic of China

${ }^{5}$ Institute for Fusion Studies, University of Texas at Austin, Austin, TX 78712, United States of America

"Corresponding author: zychen@hust.edu.cn

\begin{abstract}
The J-TEXT tokamak has been operated for 10 years since its first plasma obtained at the end of 2007. The diagnostics development and the main modulation systems, i.e. resonant magnetic perturbation (RMP) systems and the massive gas injection (MGI) systems, will be introduced in this paper. Supported by these efforts, J-TEXT has contributed to research on several topics, especially on the RMP physics and disruption mitigation. Both experimental and theoretical
\end{abstract}


research shows that the RMP could lock, suppress or excite the TM, depending on the RMP amplitude, the frequency difference between RMP and rational surface rotation, and the initial stabilities. The plasma rotation, particle transport and operation region are influenced by the RMP. Utilizing the MGI valves, disruptions have been mitigated with pure $\mathrm{He}$, pure $\mathrm{Ne}$, and a mixture of He and $\operatorname{Ar}(9: 1)$. A significant runaway current plateau could be generated with moderate amounts of argon injection. The RMP has been shown to suppress the generation of runaway current during disruptions.

Keywords: J-TEXT, resonant magnetic perturbation, disruption

(Some figures may appear in colour only in the online journal)

\section{Introduction}

The Joint-TEXT (J-TEXT) tokamak [1, 2] is a conventional iron core tokamak reconstructed from the Texas EXperimental Tokamak Upgrade (TEXT-U) [3]. The J-TEXT is operated at a major radius $R_{0}=1.05 \mathrm{~m}$, minor radius $a=25-29 \mathrm{~cm}$ with a movable titanium-carbide-coated graphite limiter. The typical J-TEXT discharge in the limiter configuration is done with toroidal field $B_{\mathrm{t}}$ of $\sim 2.0 \mathrm{~T}$, plasma current $I_{\mathrm{p}}$ of $\sim 200 \mathrm{kA}$, pulse length of $800 \mathrm{~ms}$, plasma densities $n_{\mathrm{e}}$ of $(1-7) \times 10^{19}$ $\mathrm{m}^{-3}$, and electron temperature $T_{\mathrm{e}}$ of $\sim 1 \mathrm{keV}[4]$.

The J-TEXT tokamak has been operated for 10 years since its first plasma obtained at the end of 2007 [2]. During the past decade, J-TEXT has served as the only medium-sized tokamak in Chinese universities. With the great efforts to develop several diagnostics and to construct a few modulation systems, J-TEXT has contributed to research on several topics [1, 2, 4-6], including turbulence, transport, the interaction between resonant magnetic perturbations (RMPs) and tearing modes (TMs), and the disruption, etc. This paper will briefly summarize the key results on RMP 
and disruption physics in the last 10 years from J-TEXT. In section 2, the diagnostic development, the RMP system, the massive gas injection (MGI) systems are described; in section 3, the impacts of RMP on TM instabilities are described; in section 4, the studies on disruption physics are described. The last section is a summary.

\section{Experimental setup}

\subsection{Diagnostic development}

The diagnostics of a tokamak play key roles in operating the plasma and carrying out the physics studies. On J-TEXT, most of the fundamental diagnostics have been operating since its first plasma in 2007. Moreover, from that time several new diagnostics were developed and upgraded on J-TEXT for physics studies, especially focusing on the MHD activity and disruption mitigation. This subsection summarizes the current status of diagnostics on J-TEXT.

The plasma parameters are measured with spatial and time resolution as high as possible, so that both the equilibrium evolution and the MHD activity could be resolved. The electron density and current profiles are measured by the three-wave FIR laser polarimeter-interferometer system (POLARIS) with high phase resolution $\left(<0.1^{\circ}\right.$ for Faraday angle and $<1^{\circ}$ for line-integrated density at $50 \mathrm{kHz}$ bandwidth), high temporal resolution (< $1 \mu \mathrm{s})$ and high spatial resolution (15 $\mathrm{mm}$ minimum) [7]. The electron temperature profile is measured by a 24-channel heterodyne electron cyclotron emission (ECE) radiometer, covering most of the plasma within the frequency range $80-125 \mathrm{GHz}$ [8]. The radial profiles of ion temperature and toroidal rotation are measured by two spectrometer systems, i.e. the X-ray imaging crystal spectrometer (XICS) [9] for helium-like argon $\left(\mathrm{Ar}^{16+}\right)$ in the core and the high-resolution ultraviolet/visible spectrometer [10] for $\mathrm{C}^{2+}, \mathrm{C}^{4+}, \mathrm{O}^{4+}$ in the edge. 
Several arrays have been developed for the radiation measurements, including the CIII (465 $\mathrm{nm}), \mathrm{H}_{\alpha}(656 \mathrm{~nm})$, Absolute eXtreme UltraViolet (AXUV), soft X-ray (SXR) and hard X-ray (HXR). Two 18-channel photodiode arrays are installed to inspect the CIII (impurity source at edge)/ $\mathrm{H}_{\alpha}$ (edge recycling) from both high field side (HFS) and low field side (LFS) [1]. The SXR are measured by five 16-channel arrays located at the same toroidal position [11]. Three arrays view the internal plasma region $(r / a<0.7)$ with a spatial resolution about $2 \mathrm{~cm}$ for tomography. The other two arrays view the plasma core and boundary respectively, with higher spatial resolution $1 \mathrm{~cm}$ for monitoring local MHD activity. The poloidal distribution of AXUV radiation is measured by 4 arrays located at a single toroidal position [12], while 4 arrays and 8 single-channel detectors are toroidally distributed at the same poloidal location for measuring the toroidal distribution [13], especially the toroidal asymmetry of radiation during the disruption. The spatial distribution of HXR is measured by several detectors [14], including $7 \mathrm{NaI}$ scintillators (energy range $0.5-5 \mathrm{MeV}$ ) arranged on the equatorial plane and $16 \mathrm{CdTe}$ semiconductor detectors (Energy range $30 \mathrm{keV}-1 \mathrm{MeV}$ ) at different toroidal and poloidal positions. These HXR arrays serve as a powerful tool for detecting the loss of runaway electron with toroidal and poloidal resolution.

In addition to the 1D measurement, two cameras are also installed to record the 2D images of the plasma radiation. The fast frame visible camera (frame size $1024 \times 1024$, frame rate $10-900$ $\mathrm{kHz}$ ) has been used to monitor the penetration of impurities inside the plasma [15], while the infrared camera working in the wavelength of 3-5 $\mu \mathrm{m}$ has been developed to view the runaway electrons, approaching several tens of $\mathrm{MeV}$, by synchrotron radiation [16]. ,

The perturbed magnetic fields due to MHD activity are measured by 4 Mirnov arrays [17] 
and 1 saddle loop array $[18,19]$. The Mirnov arrays consist of 2 poloidal arrays measured at $48+$ 24 poloidal locations respectively and 2 toroidal arrays measured at $16+9$ toroidal locations. In each location, both the poloidal and the radial magnetic fields $\left(B_{\mathrm{p}}\right.$ and $\left.B_{\mathrm{r}}\right)$ are measured. The saddle loop array, containing 12 toroidally distributed saddle loops, is used to measure the static/quasi-static non-axisymmetric radial magnetic field due to the plasma response to RMP, e.g. the locked mode.

\subsection{RMP system}

To study the RMP physics, two sets of magnetic perturbation coils have been constructed on J-TEXT, as shown in Figure 1 [20]. One is reconstructed from TEXT-U [21], and referred to as static RMP (SRMP), and the other, dynamic RMP (DRMP) is newly designed and installed for producing rotating RMPs.

The SRMP coils consist of three cosine coils with six current loops and two sine coils with five current loops, as shown in Figure 1(b). They are installed outside the vacuum vessel wall under the toroidal field coils. The cosine coils produce dominant $m / n=2 / 1$ and $3 / 1$ RMP components with the amplitude of 0.54 and 0.38 Gauss $/ \mathrm{kA}$ at the plasma edge, where $m$ and $n$ are the poloidal and toroidal mode number. The spectrum produced by the sine coils is dominated only by the $3 / 1$ component with 0.38 Gauss/kA. Normally, they are connected in series and fed with a DC pulsed power supply.

The DRMP coils consist of 12 saddle coils, which are divided into four groups and placed at four equally-spaced toroidal locations as shown in Figure 1(a). Each group has three coils located at the top, low field side, and bottom of the vessel, as shown in Figure 1(c). The DRMP coils can produce a dominant $2 / 1$ component of 2.56 Gauss $/ \mathrm{kA}$ or $3 / 1$ component of 1.2 Gauss $/ \mathrm{kA}$ by 
adjusting the coil connections. The DRMP coils are installed inside the vacuum vessel wall, so that they can produce high frequency radial magnetic fields with considerable amplitudes. By feeding AC currents to the DRMP coils with proper phases, a 2/1 RMP rotating at a few kilohertz, e.g. 1-6 $\mathrm{kHz}$, can be produced with the amplitude around 0.5 Gauss $/ \mathrm{kA}$.

The RMP could rotate in either electron or ions diamagnetic directions. In the J-TEXT Ohmic plasmas, the tearing mode is observed to rotate only in the electron diamagnetic direction. So the RMP was either static or rotating in the electron diamagnetic directions in this paper.
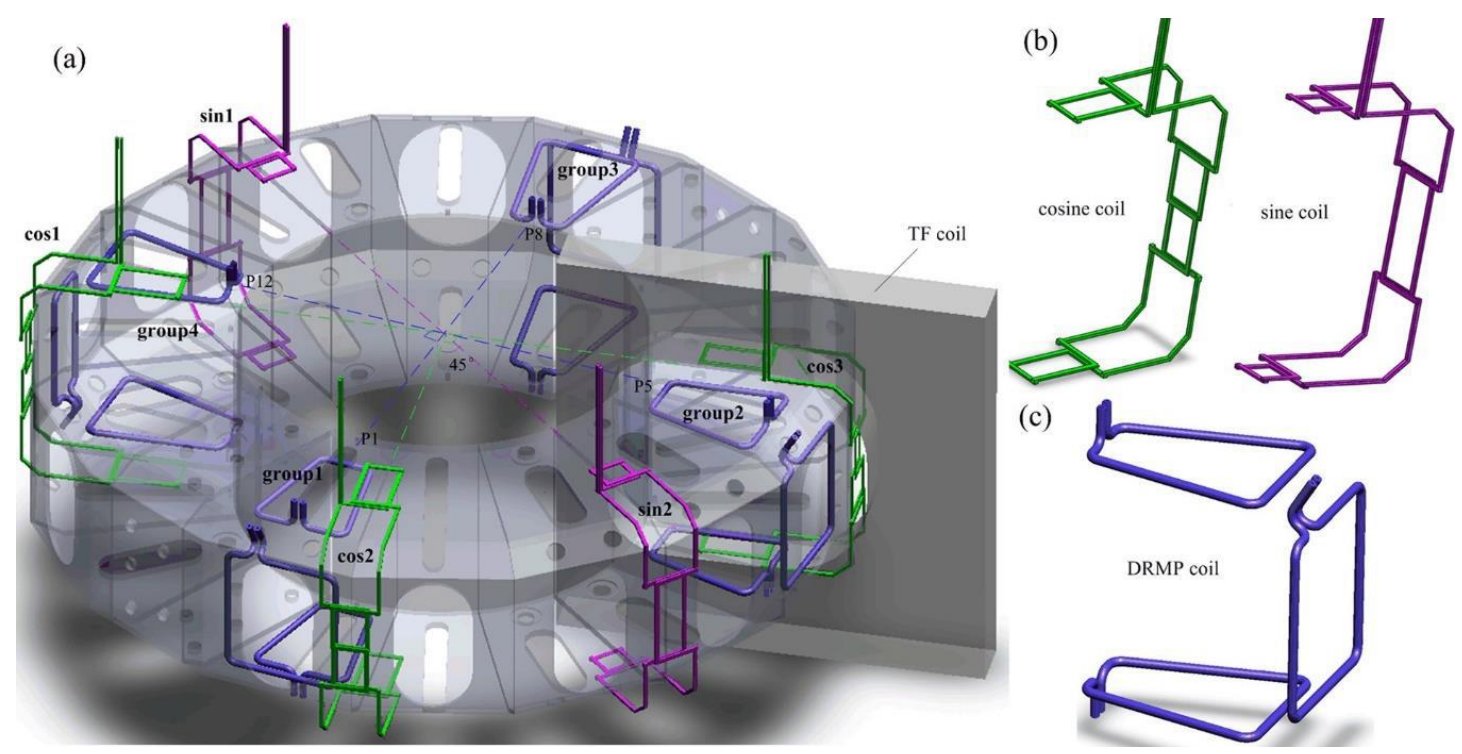

Figure 1. (a) Layout of RMP coils on J-TEXT, (b) the structure of the SRMP coils (cosine and sine coils) and (c) the DRMP coils. Reprinted from [20], Copyright (2014), with permission from Elsevier.

Three sets of power supplies were built for the RMP coils on J-TEXT. The SRMP coils were fed with a DC power supply (PS) reconstructed from TEXT-U, while the DRMP coils were fed with either two DC PSs or two AC PSs. The DC PSs for DRMP coils can produce a maximum current of $7 \mathrm{kA}$ with a pre-set waveform [22]. The two AC PSs use an AC-DC-AC converter including a series resonant inverter that can generate a maximum current of $5 \mathrm{kA}$ in the frequency range from $800 \mathrm{~Hz}$ up to $6 \mathrm{kHz}[23,24]$. 


\subsection{MGI system}

Two MGI valves have been developed for the plasma fast shutdown experiments on the J-TEXT tokamak [25]. They are based on the eddy-current repulsion mechanism. The piston of the MGI valve is made of non-ferromagnetic material so that it can be installed as close as the vacuum vessel. The valve is $0.6 \mathrm{~m}$ away from the plasma boundary. A main feature of the MGI valve is that the coils are installed separately from the mushroom cap of the piston and connected with the atmosphere. Thus the temperature of the coils can be kept to low values. The pulse current is produced by a discharge circuit which is triggered by the central control system. A high speed camera has been used to calibrate the moving distance of the piston. It shows that the reaction time of the MGI valve is about $0.3 \mathrm{~ms}$. The MGI valve can remain open for $\sim 10 \mathrm{~ms}$. The maximum density of the injected purities is more than 100 times that of the plasma inventory. A $30 \mathrm{ml}$ MGI valve has been installed at the bottom port of No. 9. It can be operated in the range of 5-30 bar. Another $60 \mathrm{ml}$ MGI valve was installed at upper port of No.9. It can be operated in the range of $5-40$ bar.

\section{Interaction between RMP and tearing mode}

Recent J-TEXT research has contributed to the understanding of the interaction between RMP and tearing mode instabilities. This part summarizes the main findings, including the locking [26-34], suppression [26-29], excitation [35] of the tearing mode and the corresponding thresholds of RMP. The modelling results described in this section were carried out by solving the nonlinear MHD equations using the initial value code TM1 [36, 26].

In a low beta plasma with tearing mode, the external RMP field leads to two main effects [37, 26], i.e. applying a resonant electromagnetic torque (Equation (1)) and modifying the tearing 
stability index (Equation (2)), as follows:

$$
\begin{aligned}
& \left\{\begin{array}{l}
T_{\theta \mathrm{EMs}}=-4 \pi^{2} R_{0} \frac{m^{2}}{\mu_{0}}\left|\Psi_{\text {vac }}\right| \Psi \mid \sin (\Delta \xi) \\
T_{\varphi \mathrm{EMs}}=-\frac{n}{m} T_{\theta \mathrm{EMs}}
\end{array}\right. \\
& \Delta_{\mathrm{RMP}}^{\prime}=\frac{2 m}{r_{s}}\left(\frac{W_{\mathrm{vac}}}{W}\right)^{2} \cos (\Delta \xi) \\
& \Delta \xi(t)=\int_{0}^{t} \omega\left(t^{\prime}\right) \mathrm{d} t^{\prime}+\arg \left(\Psi_{\mathrm{vac}}\right)-\arg (\Psi)
\end{aligned}
$$

where $\left|\Psi_{\mathrm{vac}}\right|$ is the helical flux induced by RMP at $q=m / n$ surface in the absence of a plasma, and it is proportional to the RMP amplitude. $|\Psi|$ is the reconnected flux. $W$ is the full island width and $W_{\text {vac }}$ is the vacuum island width, which are proportional to the square root of $|\Psi|$ and of $\left|\Psi_{\text {vac }}\right|$, respectively. $\Delta \xi$ is the phase difference between the TM and the RMP. The $\omega$ is the circular frequency difference between the RMPs and TM.

It is shown from (Equation (1)) and (Equation (2)) that the impact of RMP on tearing mode depends strongly on the phase difference between them, $\Delta \xi$. With $0<\Delta \xi<\pi$, RMP applies a decelerating torque, while it accelerates the TM with $-\pi<\Delta \xi<0$. The RMP stabilizes (destabilizes) the TM when $\pi / 2<\Delta \xi<3 \pi / 2(-\pi / 2<\Delta \xi<\pi / 2)$.

For a rotating tearing mode, the applied static RMP would lead to the oscillation of island width and rotation due to the change of $\Delta \xi$ from 0 to $2 \pi$, as shown in Figure 2 by nonlinear MHD modelling [26]. The modelling results show that the TM spends a slightly longer time in the stabilizing phase (deceleration phase) than in the destabilizing phase (acceleration phase), contributing to a net stabilizing and braking effect on the TM. These two effects play the key role in the impact of RMP on TM as will be shown in sections 3.1 and 3.2. 
Figure 2. Time evolution of (a) normalized island width $w / a$, (b) mode angular frequency $\omega \tau_{\mathrm{R}}$, and (c) phase difference between RMP and TM from a nonlinear MHD modelling using TM1 code. Reprinted from [26], with the permission of AIP Publishing.

\subsection{Mode locking}

The mode locking on J-TEXT has been described in several papers from both experimental and theoretical point of views [26-34]. We summarize the mode locking phenomenon by displaying two discharges with different phases of the applied RMPs, as shown in figure 3.

With the ramp-up of RMP amplitude (figure 3(a)), the 2/1 tearing modes were decelerated (figure 3(b1) and (b2)) and locked in both discharges. Once the TM was locked, the Mirnov signal reduced to zero (figure $3(\mathrm{~b})$ ), the static $n=1$ radial magnetic field, $b_{\mathrm{r}}{ }^{n=1}$, increased significantly (figure 3(c)), the edge toroidal rotation of impurity changed towards co- $I_{\mathrm{p}}$ direction to about 15 $\mathrm{km} / \mathrm{s}$ (figure 3(e)) and the oscillation of electron temperature disappeared (figure 3(f) and $(\mathrm{g})$ ). The locked tearing mode rotated again after the removal of RMP. During the locked mode stage (e.g. $t$ $=0.35 \mathrm{~s}$ ), the TMs were locked at two locations with the measured $b_{\mathrm{r}}{ }^{n=1}$ phase, $\varphi^{n=1}$, equaling $15^{\circ}$ and $148^{\circ}$ in discharges \#1033209 and \#1032491 respectively. The helical phases, defined by $\xi=$ $m \theta+n \varphi$, of the O-points of the locked modes were $\xi_{\mathrm{O}}=105^{\circ}$ and $238^{\circ}$ respectively. Since $\xi_{\mathrm{ECE}}=90^{\circ}$ 
the ECE would measure near the $\mathrm{O}$ and $\mathrm{X}$ points of the locked islands respectively, which were confirmed by the radial profile of $T_{\mathrm{e}}$ without (figure 3(f)) and with (figure $3(\mathrm{~g})$ ) gradient near the $2 / 1$ rational surface $(R \sim 88 \mathrm{~cm})$.
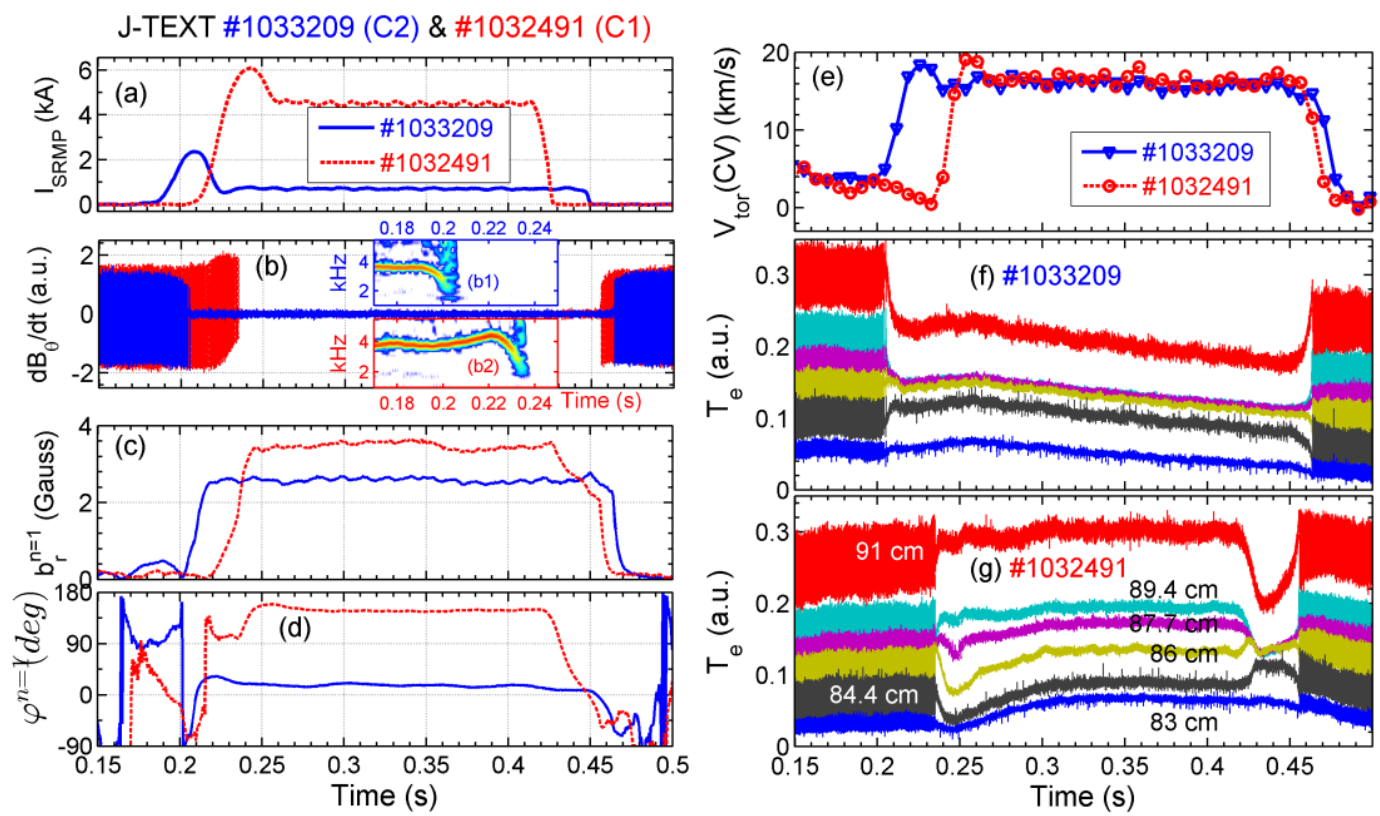

Figure 3. The locking of tearing mode in two discharges with different phases of the applied RMPs, with (a) RMP amplitude, (b) Mirnov signal, (b1) and (b2) the spectrum of Mirnov signal, (c) the amplitude and (d) the phase of $n=1$ radial magnetic field measured by locked mode detector, (e) toroidal rotation of $\mathrm{CV}$ in the edge, the electron temperature in discharges (f) \#1033209 and (g) \#1032491.

In addition, the application of a RMP field rotating at a few kilohertz could lead to the phase locking between rotating RMP and the TM [32]. The TM could either be accelerated [32] or decelerated [33], if the initial slip frequency $f_{\mathrm{s}}=f_{\mathrm{TM}}-f_{\mathrm{RMP}}$ is negative or positive. Unlike the density decrease for TM locked to static RMP [31], the particle confinement could be improved [34] if TM were accelerated as shown in Figure 4. Numerical results based on nonlinear two-fluid equations approximately agree with experimental ones, and show that the change of the electron density gradient due to torque balance is responsible for the improved particle confinement. These results reveal the role of relative rotation between the electron fluid and RMPs in affecting the particle transport. 


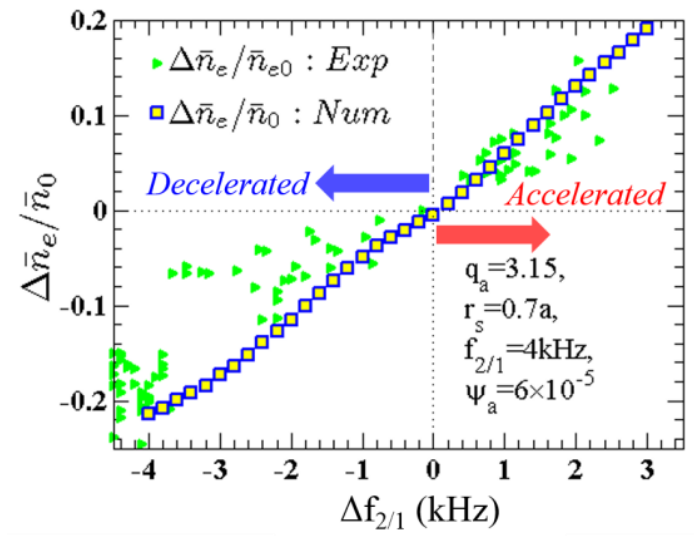

Figure 4. Relative change in line-averaged density $\Delta \bar{n}_{\mathrm{e}} / \bar{n}_{\mathrm{e} 0}$ at plasma core versus the frequency difference $\Delta f_{2 / 1}=f_{\mathrm{RMP}}-f_{2 / 1}$ after the locking of TM to rotating RMPs. The experimental and numerical results are shown by green triangles and blue squares.

\subsection{Mode suppression}

The TM could also be suppressed by the RMP field [26-29]. The J-TEXT discharge \#1030164 is displayed in Figure 5 as an example. The initial TM rotated at around $6 \mathrm{kHz}$ (Figure 5(c)). From $0.22 \mathrm{~s}$, the RMP was applied by feeding the DRMP coils with $5 \mathrm{kA}$ current. The Mirnov signal decreased with the increase of RMP amplitude, and it was completely suppressed into noise level at $0.236 \mathrm{~s}$, as shown in Figure $5(\mathrm{~b})$. The mode frequency, $f_{\mathrm{TM}}$, decreased from $6.5 \mathrm{kHz}$ to $4.1 \mathrm{kHz}$ at $t=0.232 \mathrm{~s}$, but it increased until $0.236 \mathrm{~s}$ when the TM disappeared (Figure 5(c)). The reduction of $f_{\mathrm{TM}}$ is consistent with the net braking effect of RMP, while the increase of $f_{\mathrm{TM}}$ might be due to the fact that the $T_{\mathrm{EM}}$ decreased to a weaker value with the decreasing island width [26]. Accompanying the mode suppression, the core $T_{\mathrm{e}}$ increased and sawtooth oscillations appearanced (Figure 5(d)), demonstrating the improvement of plasma confinement. After removing the RMP at $0.4 \mathrm{~s}$, the $2 / 1 \mathrm{TM}$ did not appear again (not shown here) while the large sawtooth oscillations remained in the core, indicating a reliable suppression of tearing mode. 
J-TEXT, \#1030164

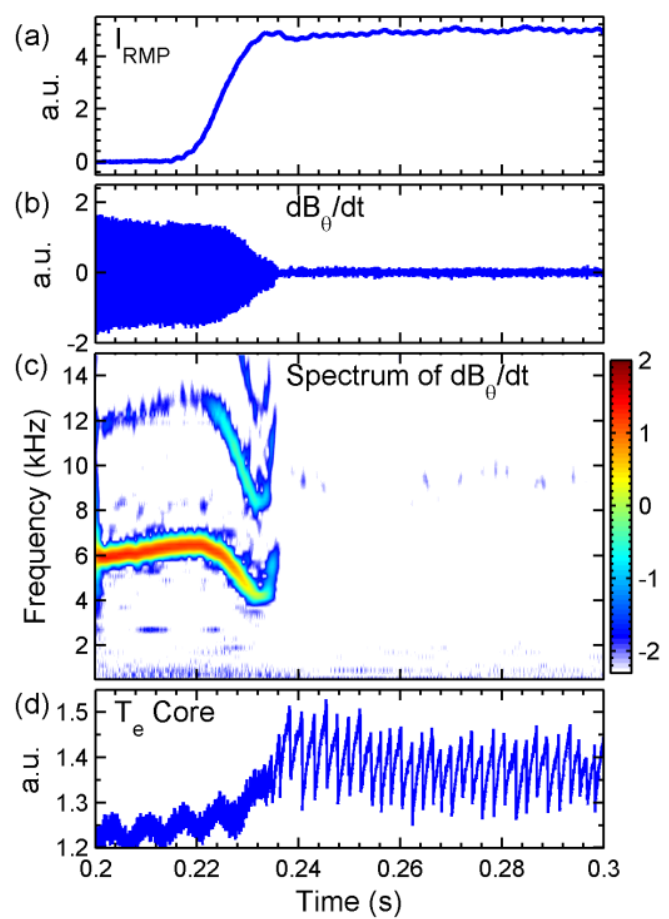

Figure 5. Complete suppression of TM. Time evolutions of (a) RMP coil current, (b) Mirnov signal $\mathrm{d} B_{\theta} / \mathrm{d} t$, (c) wavelet spectrum of $\mathrm{d} B_{\theta} / \mathrm{d} t$ and (d) the core electron temperture $T_{\mathrm{e}}$.

Although the RMP contributes a net stabilizing effect on the TM, it still remains to identify what the dominant stabilizing effect for the mode suppression is. The island width evolution equation has been derived in reference [38] as below

$$
\Delta_{\mathrm{t}}=\Delta_{0}+\Delta_{\text {sat }}+\Delta_{\mathrm{RMP}}+\Delta_{\text {ion }}
$$

where $\Delta_{\mathrm{t}}$ represents the total stability index, $\Delta_{0}$ represents the standard stability index, $\Delta_{\text {RMP }}$ represents the RMP effect, $\Delta_{\text {sat }}$ is due to the nonlinear saturation, $\Delta_{\text {ion }}$ is the destabilizing term contributed by ion polarization current. Since the mode suppression has been reproduced by the nonlineaer modelling, it is reasonable to calculate each term in Equation (4) using the analytical expression listed in reference [38] and the parameters from the modelling [26]. The integral of stability indices directly represents the contribution of these effects on the evolution of island width. As shown in Figure 6, the integral of $\Delta_{\text {RMP }}$ is always negative and much more negative than 


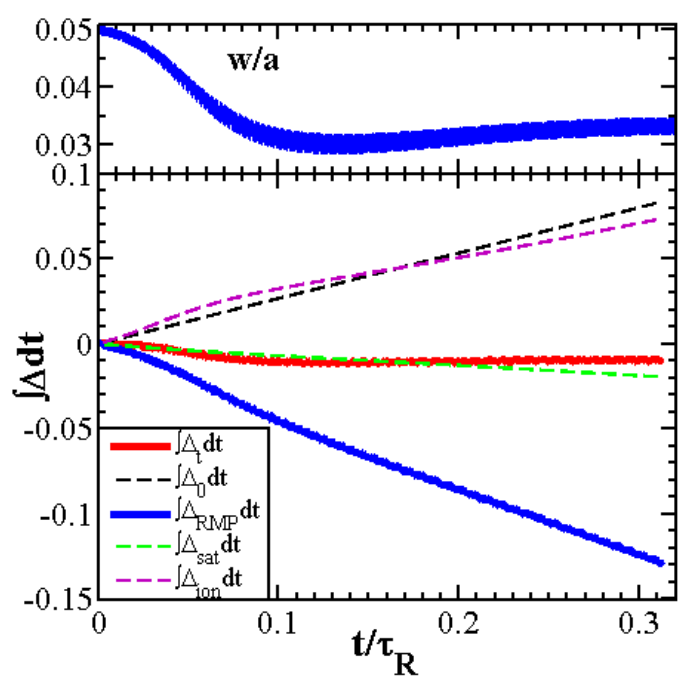

Figure 6. Time evolution of the island width and the integral of stability indexs due to several effects. The applied RMP contributes a dominant stabilizing effect on TM. Reprinted from [26], with the permission of AIP Publishing.

Utilizing this effect, the RMP has been successfully applied to suppress the disruption precursors and hence to extend the operation region towards higher density and lower $q_{\mathrm{a}}$ in J-TEXT $[39,40]$.

\subsection{Mode excitation}

In a tearing stable discharge, the application of strong RMP can lead to the excitation of locked tearing modes [35], as shown in Figure 7. In this discharge, the plasma was steadily operating with the edge safety factor $q_{\mathrm{a}}=3.5$ and without an obvious tearing mode. The 2/1 RMP was applied from $0.24 \mathrm{~s}$ by feeding current to the in-vessel DRMP coils (blue solid line in Figure 7(a)). The $n$ $=1$ plasma response, $b_{\mathrm{r}}{ }^{n=1}$ (red dashed line in Figure 7(a)), increased slowly with the ramping up of RMP current. As shown in Figure 7(b), the measured phase of plasma response ( $\xi_{\text {Sadd }}^{n=1}$, red solid line) was almost opposite to the phase of the $2 / 1 \mathrm{RMP}\left(\xi_{\mathrm{RMP}}^{n=1}\right.$, red solid line), indicating the plasma screened the external 2/1 RMP. When the $I_{\mathrm{DRMP}}$ reached its flattop at $0.31 \mathrm{~s}, b_{\mathrm{r}}{ }^{n=1}$ increased 
suddenly with $\xi_{\text {Sadd }}^{n=1}$ varying simultaneously. At the new steady state (e.g. $\left.0.35 \mathrm{~s}\right), b_{\mathrm{r}}^{n=1}$ was around 2.2 Gauss and $\xi_{\text {Sadd }}^{n=1}$ was around $50^{\circ}$. The phase of plasma response approached that of 2/1 RMP field, with $15^{\circ}$ phase lead in the plasma rotation direction (i.e. $-\xi$ direction). These show a large static $n=1$ magnetic island was excited and its phase was locked to the $2 / 1$ RMP, i.e. a locked mode was excited. This bifurcation process of the plasma response from screening to mode excitation is termed as RMP field penetration or mode penetration.

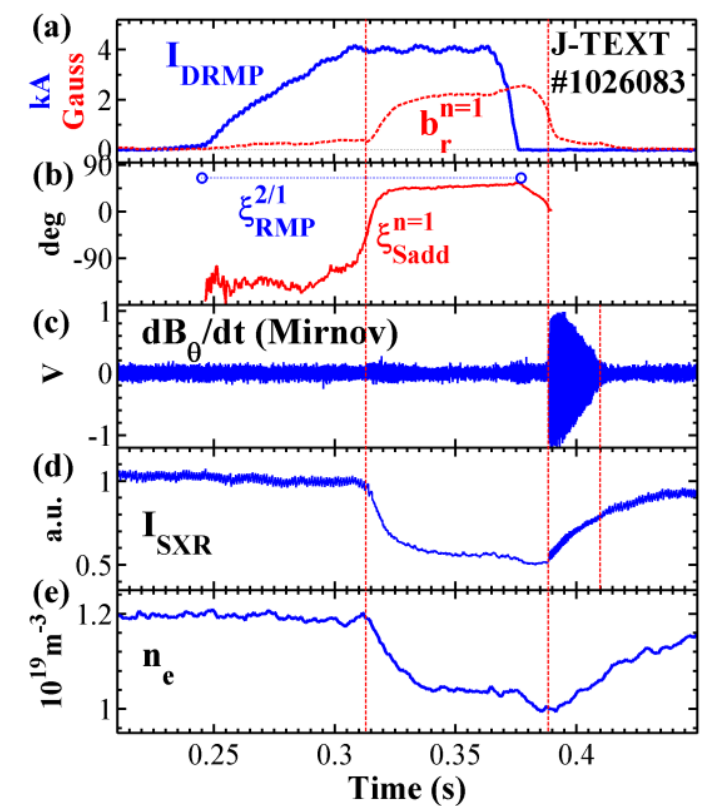

Figure 7. The excitation of a locked tearing mode in discharge \#1026083 due to the penetration of RMP field.

After the mode excitation, the sawtooth oscillations disappeared completely as shown from the SXR emission signal in Figure 7(d). Subsequently, the central line-averaged electron density $n_{\mathrm{e}}$ and the intensity of the SXR signal $I_{\text {SXR }}$ dropped significantly, indicating a substantial degradation of plasma confinement. The locked mode decreased and unlocked after the removal of RMP. The tearing mode spun up and decayed away within $20 \mathrm{~ms}$. According to the mode analysis, this rotating mode was identified to be $m / n=2 / 1$, which is the major resonant component of the applied RMP. Once the island unlocked, the signals of $I_{\mathrm{SXR}}$ and $n_{\mathrm{e}}$ began to recover towards their original values. After the island eventually vanished, the sawtooth oscillations reappeared at $t=$ 
$0.41 \mathrm{~s}$

The rotation variation plays a key role in the bifurcation from screening phase to RMP field penetration, as shown in Figure 8 for discharge \#1030179. The amplitude of RMP reached its flattop at $0.25 \mathrm{~s}$, but the RMP penetrated $0.02 \mathrm{~s}$ later. During the $0.02 \mathrm{~s}$ time delay, there was a slow change of the toroidal rotation near 2/1 rational surface (RS), indicated by $V_{\Phi}(\mathrm{CV})$ in Figure 8(d), towards the co- $I_{\mathrm{p}}$ direction. This change is consistent with the braking effect [41] of the RMP on the electron fluid, which rotates mainly in the counter- $I_{\mathrm{p}}$ direction on J-TEXT. Once the electron fluid was braked to a certain value, the RMP penetrated at $0.27 \mathrm{~s}$ and excited a large locked TM. Coinciding with the mode locking, the particle confinement was degraded [31] and the toroidal rotation was changed towards the direction opposite to the MHD rotation $[9,42]$ after the mode excitation. Detailed measurement of the radial profile of toroidal rotation will still be needed for understanding the penetration physics.

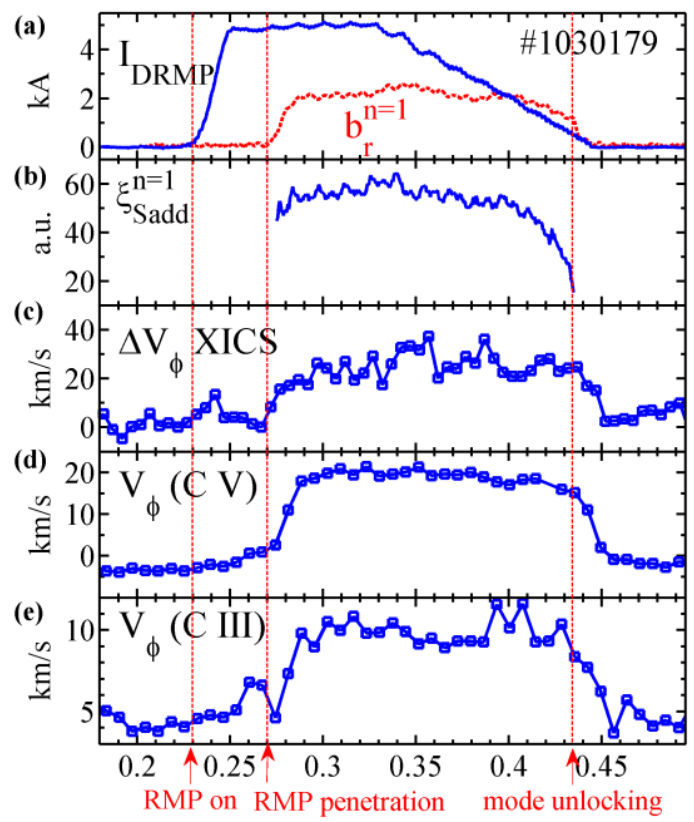

Figure 8. Time evolution of the plasma rotation at various radial locations (from the core, (c) $\Delta V_{\Phi}$ (XICS), to the edge, (e) $\left.V_{\Phi}(\mathrm{CIII})\right)$ during the mode excitation of RMP in discharge \#1030179.

As a supplement to section 3.2, a large locked mode could be excited again even if the 
unstable 2/1 tearing mode is completely suppressed by the external RMP. Figure 9 displays a discharge with a $2 / 1$ tearing mode rotating at about $8 \mathrm{kHz}$. The tearing mode was stabilized at $t=0.28 \mathrm{~s}$, with appearance of sawtooth oscillations (Figure 9(e)). The RMP was further increased from $0.305 \mathrm{~s}$ after the mode suppression. A large locked mode was excited at $0.32 \mathrm{~s}$, leading to the disappearance of sawtooth oscillations and substantial reduction in $I_{\text {SXR }}$ and $n_{\mathrm{e}}$. After the RMP was turned off, the unlocked tearing mode did not disappear as the case without initial mode.

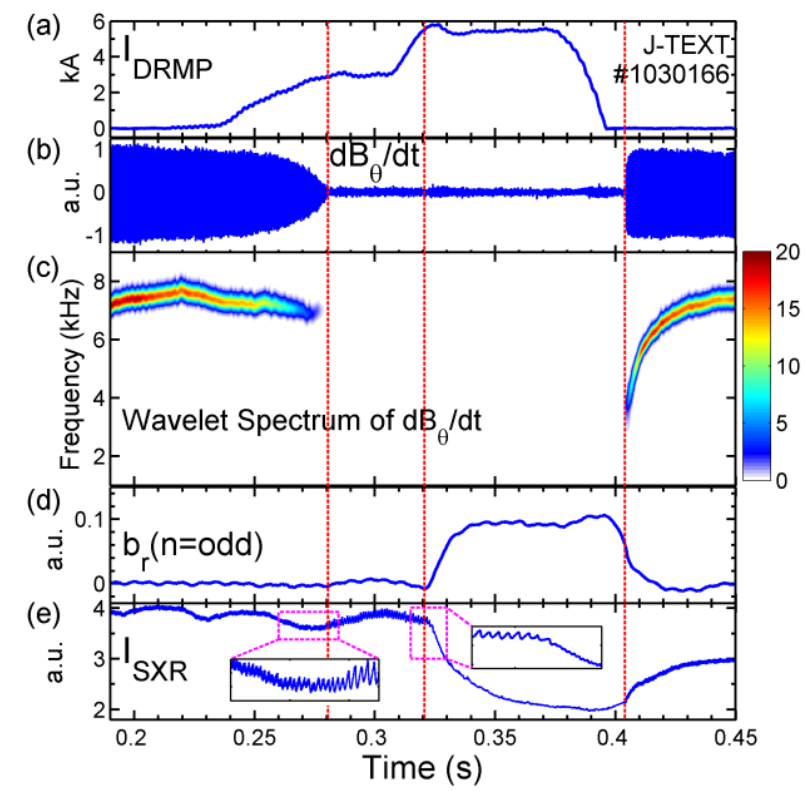

Figure 9. Mode excitation after the suppression of 2/1 tearing mode by the static RMP in discharge \#1030166. Reproduced courtesy of IAEA. Figure from [35]. Copyright (2014) IAEA.

The numerical modelling indicates that there is a small locked island (SLI) [26] before the excitation of large locked mode in Figure 8 and Figure 9. The SLI is locked at the stabilizing phase region and decouples from plasma, since its width is smaller than the viscous-resistive linear layer width. The SLI acts as a slightly non-ideal screening current due to the small resistivity, so that the RMP field is shielded within the resonant surface. The electromagnetic torque, applied by the RMP on SLI, brakes the electron fluid rotation. With a strong RMP field, the rotation braking would trigger the bifurcation from SLI to large locked island, as shown at $t=0.27 \mathrm{~s}$ in Figure $8(\mathrm{~d})$. The SLI region appears on a transient stage between the mode suppression and the large locked 
mode excitation.

\subsection{Thresholds during the interaction between RMP and tearing mode}

The RMP can influence the tearing instabilities at a very low amplitude, yet there is a clear threshold effect for the RMP to lock, suppress or excite the tearing mode, as shown in Figure 10 and Figure 11 for J-TEXT plasmas.

Figure 10 displays the TM reaction to RMP field at various mode frequencies and RMP amplitudes [29]. In the region $f_{\mathrm{MHD}}<5 \mathrm{kHz}$, the threshold for mode locking (blue squares) increases linearly with the mode frequency, while insufficient SRMP current could decelerate the TM. In the region $f_{\mathrm{MHD}} \sim 6 \mathrm{kHz}$, the RMP stronger than the threshold (black triangles) could completely suppress the TM, while the weaker RMP (red pentagrams) could only partially suppress the TM and the TM grows up again after removing RMP. Figure 10 shows that the MHD frequency played a key role in whether the RMP could lock or suppress the TM. Systematic studies by nonlinear numerical modelling have been carried out in references [26, 27, 29]. According to the modelling, the stabilizing effect of RMP is dominant over the decelerating effect and the TM could be suppressed, if the plasma has high rotation velocity, large viscosity, low Alfvén speed and small island width.

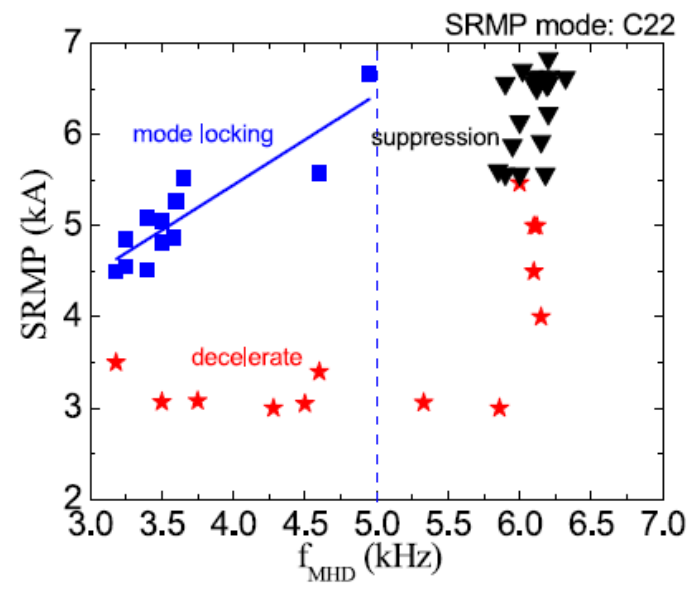


Figure 10. Dependence of the RMP threshold for mode locking (blue squares) and mode suppression (black triangles) on the MHD frequency. For RMPs weaker than the thresholds (red pentagrams), only deceleration or partical suppression could be observed. Reproduced from [29]. (C IOP Publishing Ltd. All rights reserved.

Figure 11 shows the dependence of mode penetration threshold on the electron density in J-TEXT [35]. It is found that the penetration threshold increases with increasing density with a density scaling $b_{\mathrm{r}}^{2 / 1} \propto \bar{n}_{\mathrm{e}}^{0.5 \pm 0.05}$. The nonlinear numerical modelling gives a similar density dependence with fixed rotation velocity at $q=2$ surface. This density dependence is weaker than many other devices with almost linear density dependence, yet the TEXTOR [43] and EAST [44] results also show a scaling similar to J-TEXT. Since the ITER start-up phase will operate at low density, further investigation of the mode penetration is still needed to explain the discrepancy among devices.

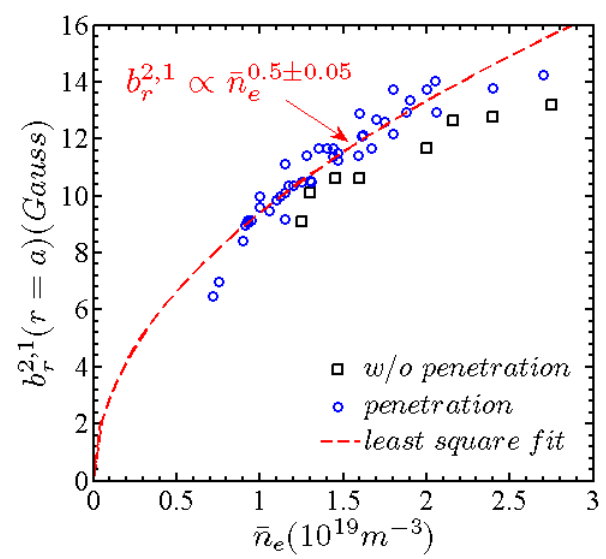

Figure 11. Dependence of mode penetration threshold on the line-averaged electron density in the core. Reproduced courtesy of IAEA. Figure from [35]. Copyright (2014) IAEA.

Since the thresholds of mode locking or mode penetration do not rely on the RMP phase, this property is usually used to measure the intrinsic error field of a device. The locked mode thresholds are measured at various RMP phases and then plotted in the polar coordinate. These thresholds should locate near a circle with shifted center, which is the measured intrinsic error field. The J-TEXT error field was measured to be around 0.31 Gauss for the $2 / 1$ component by using this technique [29]. 
Understanding of these thresholds is important for the active control of TM by using RMP. To suppress the TM, the frequency of TM should be high enough to avoid TM locking. A possible method to suppress the low frequency TMs is to apply a high frequency RMP rotating in the direction opposite to that of TM. Even for the high frequency TM, the RMP amplitude cannot be too large, since it could lead to mode excitation due to the RMP penetration as shown in Figure 9. In references [39] and [40], the application of RMP suppressed the disruption precursors and hence extended the operation region. However, the locked mode was excited at larger RMP amplitude and it caused disruption at even lower density. The operational window of RMP in TM suppression is similar to that of ELM control [45].

\section{Disruption experiment}

Avoidance and mitigation of disruptions are critical issues for advancing the tokamak concept as a viable fusion energy source. A plasma disruption in tokamaks can be considered as a sudden loss of magnetic confinement which results in a rapid, complete loss of plasma thermal and magnetic energy [46, 47]. In general, the disruption is initiated by a global instability, which occurs in two stages including thermal quench (TQ) and current quench (CQ). During plasma disruption, the thermal loads, the halo current forces and the high energy runaway electrons can cause serious damage to the tokamak [48, 49]. The magnitude of the damaging effects increases with the plasma thermal energy $W_{\text {th }}$ and magnetic energy $W_{\text {mag }}[50,51]$. Prevention and mitigation of the damage due to disruptions are essential to reliable operation of tokamaks. Studies of disruption mitigation have been carried out in J-TEXT recently.

For disruption mitigation by MGI, $\mathrm{He}, \mathrm{Ne}, \mathrm{Ar}$, and mixture gas are good candidates for disruption mitigation for different purposes. The massive injection of low $Z$ impurities has the 
advantage of slow current quench which is favorable for the suppression of runaway electrons. But its ability to radiate energy is very limited. The massive injection of high $Z$ impurities has the advantage of high efficiency for energy radiation and fast current quench which is favorable for the reduction of halo current, while it is prone to generate runaway electrons due to the high electric field induced by the fast current quench. The mixture gas injection has the advantages of both the energy radiation and runaway suppression.

The $\mathrm{He}, \mathrm{Ne}$, Ar, and mixture gas injection had been performed in the fast shutdown experiments on J-TEXT [52]. In these experiments, the target plasmas had toroidal magnetic field $B_{\mathrm{T}}=2.3 \mathrm{~T}$, the plasma current was $I_{\mathrm{p}}=160-180 \mathrm{kA}$, the line averaged electron density was about $n_{\mathrm{e}} \sim(1.0-1.2) \times 10^{19} \mathrm{~m}^{-3}$. The fast valve located at the bottom of port 9 was used in these experiments.

The temporal evolutions of plasma currents with different amounts of He injection are shown in Figure 12. Both the disruption delay time and current quench duration decreased with increasing gas injection. The current quench rate was about $12 \mathrm{MA} / \mathrm{s}$ for a small amount of the He injection. The current quench rate increased to about $46 \mathrm{MA} / \mathrm{s}$ with one order larger of gas amount. The plasmas were always runaway free due to the slow current quench for pure He MGI injection.

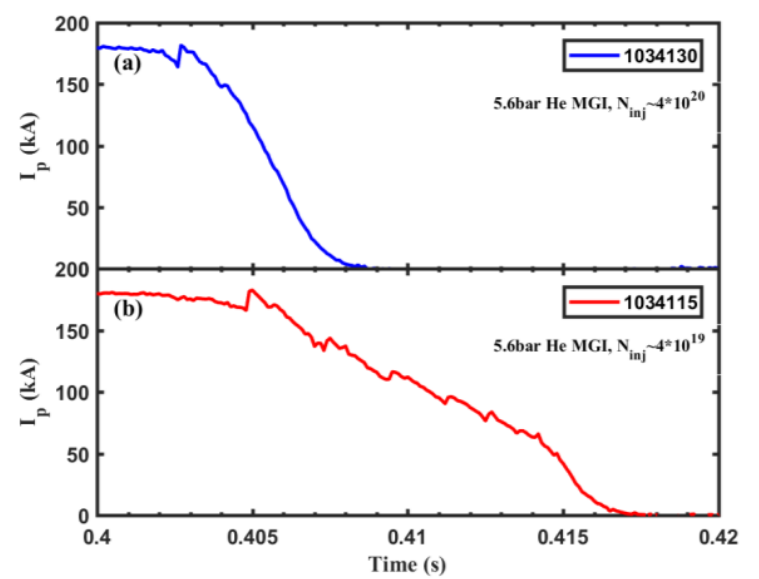

Figure 12. Fast shutdown experiments by pure He with different amount of gas injection. 
In the fast shutdown experiment with pure Ne MGI injection, the plasmas were runaway free in a large range of Ne injection also. The temporal evolutions of plasma currents with different amount of Ne injection are shown in Figure 13. The current quench rates increased with increasing gas injection. The runaway tail during disruption has not been observed. The HXR signals remained at noise level.

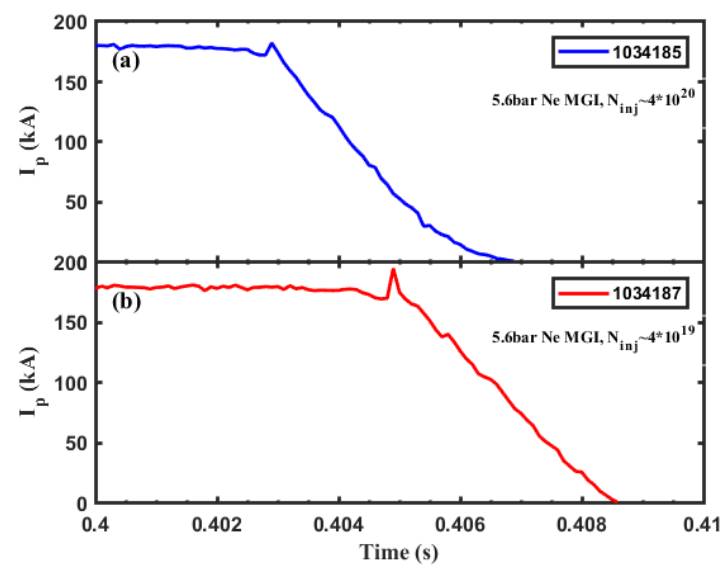

Figure 13. Fast shutdown experiments by pure Ne with different amount of gas injection.

For disruption mitigation, the method should mitigate the heat loads as well as runaway electrons at the same time. The mixture of low $Z$ impurities with high $Z$ impurities has proved to have benefits on this issue. The low $Z$ impurities contribute to the suppression of runaway electrons while the high $Z$ impurities contribute to the radiation loss of plasma energy. Fast shutdown by a mixture of He\&Ar has been performed on J-TEXT. The mixture gas consisted of $10 \% \mathrm{Ar}$ and $90 \% \mathrm{He}$. The typical waveforms of fast shutdown by mixture of $\mathrm{He} \& \mathrm{Ar}(9: 1)$ are shown in Figure 14. They were runaway free disruptions with different amounts of mixture gas injection. 


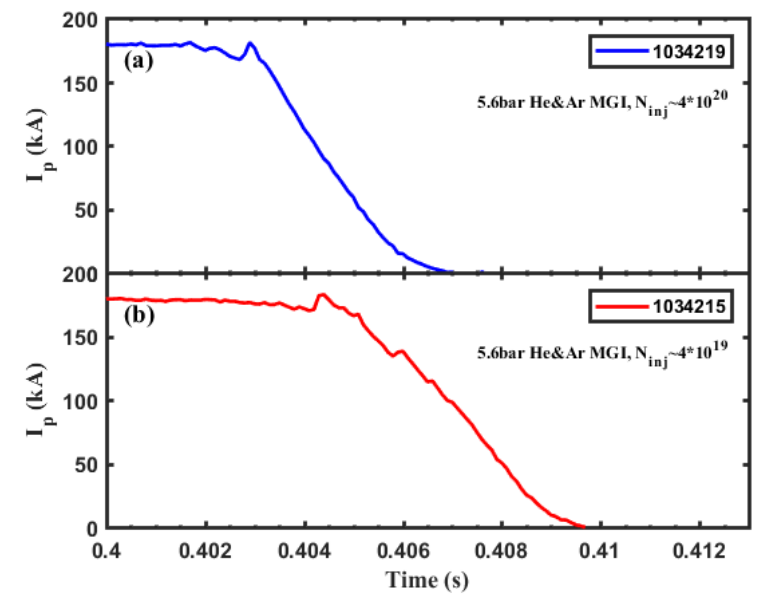

Figure 14. Typical waveforms of fast shutdown by mixture of He\&Ar (9:1).

A typical Ar injection-induced disruption with runaway current plateau is shown in Figure 15.

The fast valve was fired at $0.4 \mathrm{~s}$. The plasma disrupted in $4 \mathrm{~ms}$. In this disruption, a $110 \mathrm{kA}$ runaway current plateau was formed by Ar injection. The runaway current plateau persisted about $11 \mathrm{~ms}$ before being lost to the first wall. There was a large flux of HXR while the runaway current was built up. During the runaway current plateau, the measurement of the confined runaway beam provided significant information on runaway generation and confinement. The soft X-ray emissions during runaway current plateau offer the possibility of investigating the runaway beam generation and the beam size as a function of time [53]. The runaway electron beams produced weak X-ray images by producing $\mathrm{K}$ shell vacancies in metallic impurities in the disrupted plasma that allowed us to determine the beam structure and development by the soft X-ray emissions. The imaging of runaway beam by soft X-ray radiations for discharge \#1034059 is shown in Figure 16. According to the soft X-ray imaging, the plasma disrupted at $0.404 \mathrm{~s}$. The soft X-ray emissions increased slowly at the beginning of runaway current plateau. The runaway beam was visible from $0.406 \mathrm{~s}$ by the soft X-ray imaging. It was found that the runaway beam size was about $12 \mathrm{~cm}$. It indicated that the runaway current in the post disruption plasma had a peaked profile. 


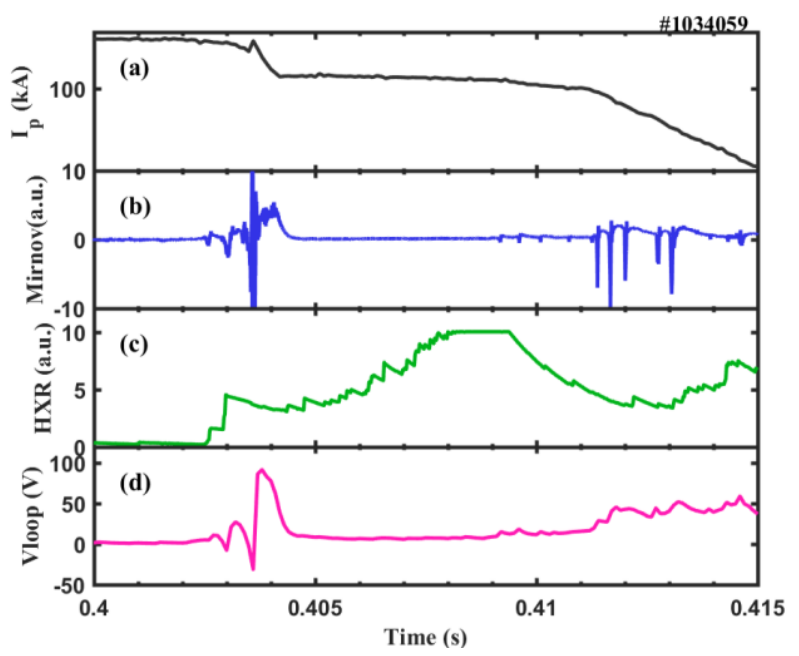

Figure 15. Fast shutdown experiments with small runaway current plateau by Ar injection.

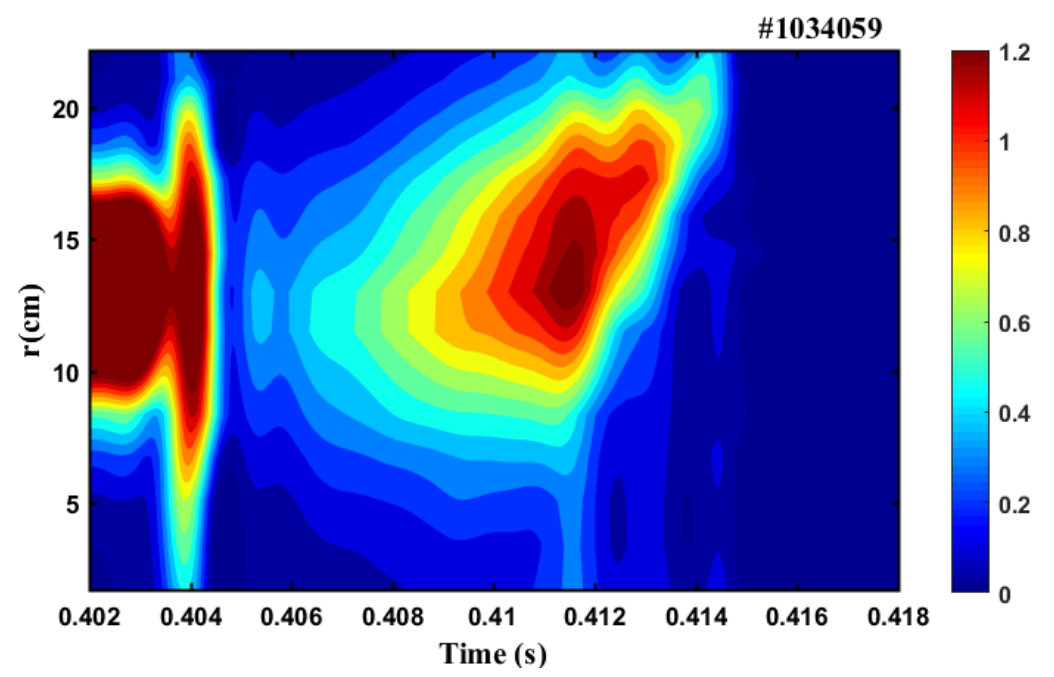

Figure 16. Soft X-ray imaging of the runaway beam during runaway current plateau for discharge \#1034059.

A large fraction of high energy runaway electrons generated during the disruption may cause severe damage to the plasma facing components and vacuum vessel. The resonant magnetic perturbation (RMP) is a powerful tool for the mitigation of ELMs. It has also been shown that the magnetic perturbation can be a potential tool for the suppression of runaway electrons [54-56]. The experimental results on TEXTOR have indicated that the Ar injection induced runaway current can be suppressed by RMP [57]. The effect of RMP on the confinement of runaway electrons has been investigated on J-TEXT [58]. The behavior of runaway electrons in a typical Ohmic discharge with $m / n=2 / 1$ RMP is shown in Figure 17. The basic parameters of target 
plasma included the plasma current $I_{\mathrm{p}}=160 \mathrm{kA}$, the toroidal magnetic field $B_{\mathrm{T}}=1.7 \mathrm{~T}$ and the central line-averaged electron density $n_{\mathrm{e}}=1.5 \times 10^{19} \mathrm{~m}^{-3}$. The burst of HXR flux at the beginning of discharge was due to the low electron density and high loop voltage. When the discharge went into flattop phase, the RMP with $m / n=2 / 1$ mode was switched on at $0.17 \mathrm{~s}$. The RMP current was 6.5 kA which corresponding to an amplitude of about 4.1 Gauss $B_{\mathrm{r}}$ at the plasma edge. It was found that the frequency of the $m / n=2 / 1$ mode characterized by the Mirnov signal gradually slowed down with an increase of RMP current. A sudden increase of runaway electron loss rate, accompanied by a sharp decrease of the amplitude of SXR signal, was observed at $0.225 \mathrm{~s}$ as indicated by the sharp increase of HXR flux in the forward direction. When the RMP was switched off, the HXR flux returned to its original level. Those findings demonstrated that the confinement and transport of runaway electrons can be significantly influenced by the RMP field.

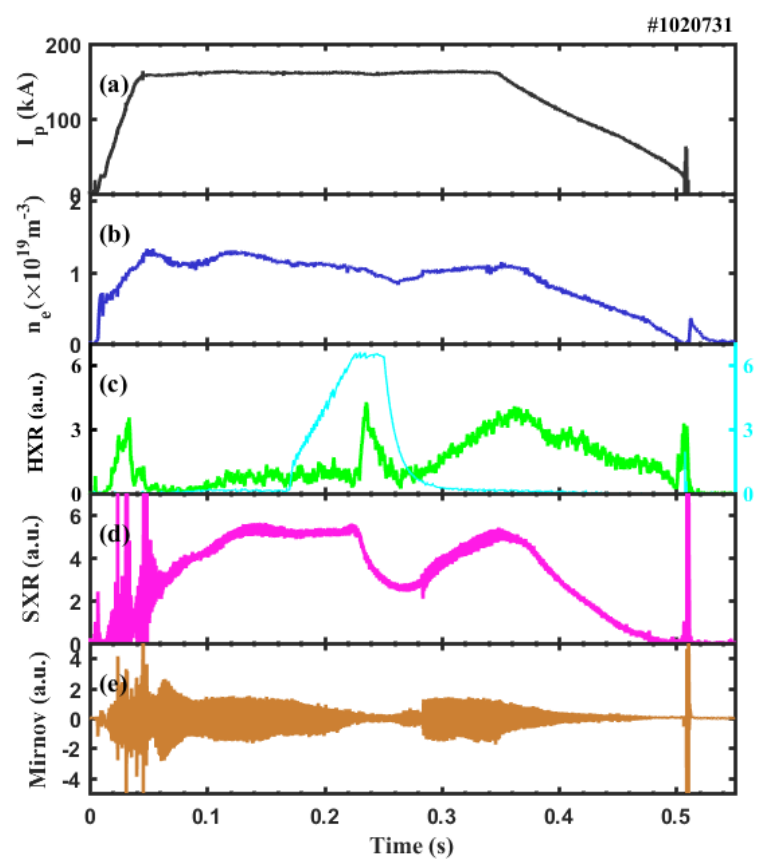

Figure 17. Waveforms of a typical Ohmic discharge (\#1020731) with RMP in the flattop phase: (a) the plasma current, (b) the center line-averaged electron density, (c) the HXR flux in the forward direction and the current of the RMP coil, (d) the soft X-ray signal, (e) the Mirnov signal .

Based on the previous studies of the enhancement of runaway electrons loss by the application of RMP during the flat top phase, the effect of RMP with $m / n=2 / 1$ mode on runaway 
generation during disruption has been investigated recently [52]. The application of RMP with $m / n$ $=2 / 1$ mode during disruption is shown in Figure 18. The target plasma had toroidal magnetic field $B_{\mathrm{T}}=2.3 \mathrm{~T}$, the plasma current was $I_{\mathrm{p}}=180 \mathrm{kA}$, the line averaged electron density was about $n_{\mathrm{e}}=$ $(1.0-1.2) \times 10^{19} \mathrm{~m}^{-3}$. The fast valve was fired at $0.4 \mathrm{~s}$ with moderate argon injection to produce a stable runaway current plateau. Without the application of RMP, the runaway current plateau in the reference discharges were lager than $60 \mathrm{kA}$. The runaway current persisted more than $12 \mathrm{~ms}$ before loss to the first wall. With the same plasma parameters and equal amount Ar injection in the discharges, 5 Gauss static RMP with 2/1 mode was applied just before the disruption. In order to prevent the generation of runaway electrons by RMP, the DRMP coils were powered from 0.38 $\mathrm{s}$ since RMP current needed about $10 \mathrm{~ms}$ to ramp up. Then the runaway current in those discharges was partially suppressed by the RMP. Both the amplitude and the length of runaway current plateau decreased. The amplitude of runaway current plateau decreased to less than $50 \mathrm{kA}$. The length of runaway current plateau reduced to less than $8 \mathrm{~ms}$. This indicated that the application of RMP can significantly reduce runaway production by enhancing runaway loss rate during disruption. All the results above suggested that the MGI and RMP are promising tools to mitigate the disruption, which are still need further understanding by experimental and theoretical studies.

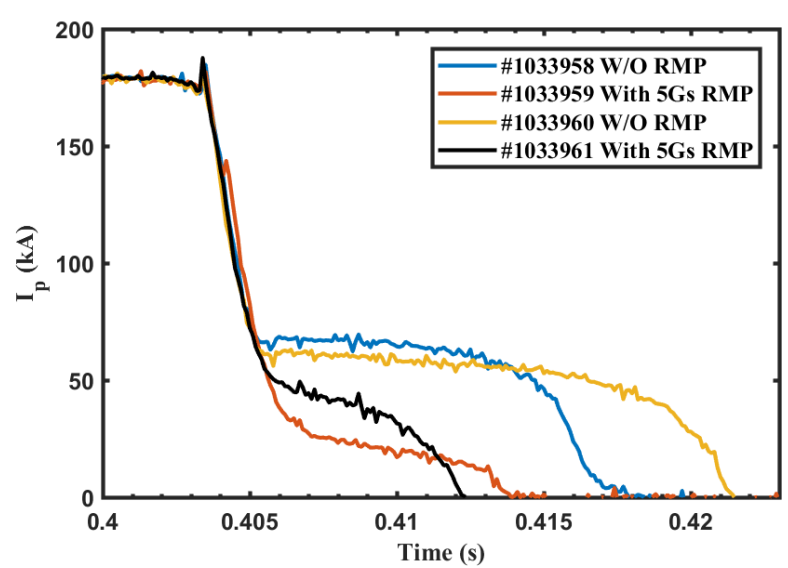


Figure 18. Waveforms of Ar induced runaway current plateau with/without the application of static RMP. The application of static RMP during disruption reduced the runaway current.

\section{Summary}

The J-TEXT tokamak has been operated for 10 years since its first plasma obtained at the end of 2007. Extensive developments of the diagnostics have been achieved, including the polarimeter-interferometer, the X-ray imaging crystal spectrometer, the spatial distribution of HXR, etc. The high frequency RMP systems and the MGI systems were constructed, supporting the study of RMP physics and disruption mitigation.

The RMP is found to change the tearing mode instabilities and to stimulate plasma response in several aspects. Both experimentally and theoretically research shows that the RMP could lock, suppress or excite the TM, depending on the RMP amplitude, the frequency difference between RMP and rational surface, and the initial stabilities. Qualitative agreement between experiments and numerical results show that RMP applied a net stabilizing and braking effect on the TM. In addition, the SLI region appears either after the mode suppression or before the mode excitation, which has been found from the numerical modelling and suggested by experimental data obtained in J-TEXT. By applying the rotating RMP, the particle transport could be improved or degraded, while the plasma rotation could either be accelerated or decelerated. The RMP could also extend the operation region of the plasma.

Disruption mitigation has been investigated on J-TEXT via both MGI and RMP. It was found that the disruption can be mitigated by the massive injection of pure $\mathrm{He}$, pure $\mathrm{Ne}$, and a mixture of He and $\operatorname{Ar}$ (9:1). However moderate amounts of argon injection could generate a significant runaway current plateau. The runaway electrons can be clearly lost during the flat top phase and during disruption by applying the RMP. 


\section{Acknowledgments}

This work is supported by the National Magnetic Confinement Fusion Science Program of China (Nos. 2014GB106001，2015GB111001，2015GB111002 and 2015GB120003) and National Natural Science Foundation of China (Nos. 11505069, 11275079 and 11405068).

\section{References}

[1] Zhuang G et al 2011 Nucl. Fusion 51094020

[2] Zhuang G et al 2009 Plasma Sci. Technol. 11439

[3] Gentle K W et al 1995 The Texas experimental tokamak: a plasma research facility DOE/ER/542-41-151, FRCR \#470 University of Texas at Austin

[4] Zhuang G et al 2013 Nucl. Fusion 53104014

[5] Zhuang G et al 2015 Nucl. Fusion 55104003

[6] Zhuang G et al 2017 Nucl. Fusion 57102003

[7] Chen J et al 2014 Rev. Sci. Instrum. 85 11D303

[8] Yang Z J et al 2016 Rev. Sci. Instrum. 87 11E112

[9] Jin W et al 2014 Rev. Sci. Instrum. 85023509

[10] Cheng Z F et al 2014 Rev. Sci. Instrum. 85 11E423

[11] Li J C et al 2014 Rev. Sci. Instrum. 85 11E414

[12] Zhang X L et al 2014 Rev. Sci. Instrum. 85 11E420

[13] Tong R H, Chen Z Y, Huang D W, et al 2018 Measurement of the toroidal radiation asymmetry during massive gas injection triggered disruptions on J-TEXT submitted to Rev. Sci. 
Instrum.

[14] Huang D W et al 2014 Rev. Sci. Instrum. 85 11D845

[15] Tang Y et al 2015 Phys. Lett. A 3791043

[16] Tong R H et al 2016 Rev. Sci. Instrum. 87 11E113

[17] Guo D J et al 2017 Rev. Sci. Instrum. 88123502

[18] Ding Y H et al 2014 Rev. Sci. Instrum. 85043502

[19] Guo D J et al 2017 AIP Adv. 7105002

[20] Rao B et al 2014 Fusion Eng. Des. 89378

[21] Craven W A 1996 Resonant external magnetic perturbations on the Texas experimental tokamak $\mathrm{PhD}$, The University of Texas at Austin, Washington, USA

[22] Zeng W B et al 2014 Plasma Sci. Technol. 161074

[23] Yi B et al 2013 Fusion Eng. Des. 881528

[24] Yi B et al 2014 Rev. Sci. Instrum. 85113501

[25] Luo Y H et al 2014 Rev. Sci. Instrum. 85083504

[26] Hu Q M et al 2013 Phys. Plasmas 20092502

[27] Hu Q M et al 2012 Nucl. Fusion 52083011

[28] Rao B et al 2013 Phys. Lett. A 377315

[29] Jin W et al 2013 Plasma Phys. Controlled Fusion 55035010

[30] Rao B et al 2013 Rev. Sci. Instrum. 84043504

[31] Hu Q M et al 2014 Nucl. Fusion 54064013

[32] Rao B et al 2013 Plasma Phys. Controlled Fusion 55122001

[33] Yan W et al 2018 Plasma Phys. Controlled Fusion 60035007 
[34] Hu Q M et al 2014 Nucl. Fusion 54122006

[35] Wang N C et al 2014 Nucl. Fusion 54064014

[36] Yu Q and Günter S 2008 Nucl. Fusion 48065004

[37] Fitzpatrick R 1993 Nucl. Fusion 331049

[38] Fitzpatrick R, Rossi E and Yu E P 2001 Phys. Plasmas 84489

[39] Hu Q M et al 2016 Plasma Phys. Controlled Fusion 58025001

[40] Huang M X et al 2016 Plasma Phys. Controlled Fusion 58125002

[41] Yu Q et al 2008 Nucl. Fusion 48024007

[42] Cheng Z F et al 2013 Rev. Sci. Instrum. 84073508

[43] Loewenbrueck K. et al 2007 Proc. 34st EPS Conf. on Plasma Physics (Warsaw, Poland, 2007) vol 31F (ECA) P-1.136 http://epsppd.epfl.ch/Warsaw/pdf/P1 136.pdf

[44] Wang H H et al 2018 Nucl. Fusion 58056024

[45] Liang Y F Edge localized mode (ELM) In: Igochine V Springer Series on Atomic, Optical, and Plasma Physics New York: Springer 2014: 143

[46] Wesson J A et al 1989 Nucl. Fusion 29641

[47] Schuller F C 1995 Plasma Phys. Controlled Fusion 37 A135

[48] Hender T C et al 2007 Nucl. Fusion 47 S128

[49] Riccardo V and JET EFDA contributors. 2003 Plasma Physics and Controlled Fusion 45 A269

[50] Martín-Solís J R et al 2014 Nucl. Fusion 54083027

[51] Zhang M et al 2015 J. Fusion Energy 341411 
[52] Chen Z Y et al 2016 Nucl. Fusion 56112013

[53] Plyusnin V V et al 2006 Nucl. Fusion 46277

[54] Yoshino R and Tokuda S 2000 Nucl. Fusion 401293

[55] Finken K H et al 2007 Nucl. Fusion 4791

[56] Finken K H et al 2006 Nucl. Fusion 46 S139

[57] Lehnen M et al 2008 Phys. Rev. Lett. 100255003

[58] Chen Z Y et al 2012 Rev. Sci. Instrum. 83056108 